\title{
Impact of gene expression data pre-processing on expression quantitative trait locus mapping Aurelie Labbe ${ }^{* \dagger 1,2}$, Marie-Paule Roth ${ }^{\dagger 3}$, Pierre-Hugues Carmichael ${ }^{1}$ and Maria Martinez ${ }^{\dagger 3}$
}

Address: ${ }^{1}$ Département de Mathématiques et de Statistique, Université Laval, Québec, G1K 7P4, Canada, ${ }^{2}$ Centre de Recherche Université Laval Robert Giffard, Québec, G1K 7P4, Canada and ' INSERM U563, Centre de Physiopathologic de Toulouse Purpan Toulouse, F-31300, France; Université Toulouse, III Paul-Sabatiere, Toulouse, F-31400, France

Email: Aurelie Labbe* - alabbe@mat.ulaval.ca; Marie-Paule Roth - roth@cict.fr; Pierre-Hugues Carmichael - pcarmich@mat.ulaval.ca; Maria Martinez - maria.martinez@toulouse.inserm.fr

* Corresponding author †Equal contributors

from Genetic Analysis Workshop 15

St. Pete Beach, Florida, USA. II-15 November 2006

Published: 18 December 2007

BMC Proceedings 2007, I (Suppl I):SI53

This article is available from: http://www.biomedcentral.com/I753-656I/I/SI/SI 53

(C) 2007 Labbe et al; licensee BioMed Central Ltd.

This is an open access article distributed under the terms of the Creative Commons Attribution License (http://creativecommons.org/licenses/by/2.0), which permits unrestricted use, distribution, and reproduction in any medium, provided the original work is properly cited.

\section{Background}

Evidence for heritability of mRNA levels has been observed in several organisms like the mouse, yeast, and human [1-3]. Therefore, it is possible to consider tran- script levels measured using DNA microarrays as quantitative traits and localize the genes controlling them by quantitative trait locus (QTL) analyses. However, one of the challenges is to remove optical noise and take into 
account nonspecific hybridization in the microarray experiments. In addition, in the Affymetrix system, each gene is represented by 11-20 Perfect Match (PM) and Mismatch (MM) pairs of probes, each probing a different region of the mRNA transcript, typically within 600 base pairs of the 3' end [4]. It is also important to find a way to combine the 11-20 probe pair intensities for a given gene into a single measure. Several data pre-processing methods are now available. Of several such proposed methods, three have commonly been employed: the Robust Multiarray Average (RMA), GeneChip RMA (GCRMA), and the Microarray Analysis Suite 5 (MAS5) methods. A detailed description of these methods can be found elsewhere [57]. These three methods convert probe-level data to expression values through the following sequence: 1) background correction, 2) normalization, and 3) summarizing the probe set values into one expression measure. Regarding the background correction step, MAS5 uses the MM probes to adjust the PM probes for probe-specific nonspecific binding [5]. On the other hand, RMA ignores the MM intensities and performs a global background correction [6]. GCRMA employs a hybrid approach and uses the probe sequence information released by Affymetrix to compute an affinity measure and describe background noise. For Step 2, RMA and GCRMA use the same normalization method based upon intensity quantiles, which imposes the same empirical distribution of intensity to each array. In MAS5, a baseline array is chosen and all the other arrays are scaled to have the same mean intensity as this array. Finally, RMA and GCRMA use the same probe summary method, based on a robust linear model. In MAS5, the probe summary is based on a robust average method.

Different DNA chip pre-processing methods have been shown to influence measures of gene expression $[6,8,9]$. Therefore, the choice of a pre-processing method significantly affects linkage results obtained in genomic analyses [10]. However, whether or not only concordant results should be further investigated is still debated (see Petretto et al. [11] and Chesler et al. [12] in their reply to Williams et al. [10]). In this paper, we focus on comparing the degree of concordance between pre-processing methods, according to the characteristics of the traits selected for linkage analysis.

\section{Methods}

The study is based on all 14 three-generation CEPH (Centre d'Etude du Polymorphisme Humain) Utah families. Gene expression levels in lymphoblastoid cells of 194 individuals have been obtained using the Affymetrix Human Focus Arrays that contain probes for 8792 transcripts. Details regarding the microarray experiments are given in Morley et al. [3].

\section{Data pre-processing}

Transcript expression data were obtained using the three methods described above: MAS5, RMA, and GCRMA. Expression levels in 82 individuals with technical replicates were averaged over replicates. All subsequent analyses were performed on $\log _{2}$-transformed values.

\section{Choice of the traits for linkage analysis}

It may be appropriate to restrict linkage analysis to the traits that are expressed in the target tissue and show detectable variation between individuals. Clearly, this is rarely the case for all the transcripts analyzed on a microarray. For example, Morley et al. [3] chose to analyze less than $50 \%$ of the measured traits, according to the ratio of the variances of trait expressions between and within individuals. Here, we first used the detection (Present/Absent) call generated by the Affymetrix MAS5 software to identify transcripts that were not reliably detected. 3727 transcripts were found to be significantly expressed in at least $80 \%$ of the arrays (at the significant level of $4 \%$ ) and are referred in this paper as the "expressed set". The other set of 5065 transcripts is referred as the "non-expressed set". Three groups of genes were selected in the expressed set of genes, based on the distribution of their expression levels measured by the MAS5 preprocessing method in the 194 available individuals: 1) the 100 genes with the highest variance in the measured expression phenotype, 2) the 100 genes with the most heritable expression, and 3) 350 genes chosen at random among the top 50\% genes with the largest variance. Note that 8 genes were common to groups 1 and 2, 22 to groups 1 and 3, and 2 to the three groups. In addition, a fourth group consisted of the 100 top most variable genes of the non-expressed set. A total of $3 \times 650$ expression phenotypes were therefore submitted to linkage analysis.

\section{Linkage analysis}

The loci controlling expression levels (the quantitative traits) of these 650 genes were localized using all autosomal marker data provided in the Problem 1 GAW15 dataset. Multipoint LOD scores were computed using the variance-component linkage test with the software Merlin [13]. In this paper, we define linkage peaks as the highest multipoint LOD within a $20 \mathrm{Mb}$-interval. In this study, we deemed as "linkage signals" linkage outcomes that have a LOD score $\geq 3$, not accounting for the multiple testing problem.

\section{Criteria to compare pre-processing methods}

Several measures were defined in order to compare the three pre-processing methods: 1) the number of linkage signals, 2) the number of traits with at least one linkage signal as well as the number of linkage signals per trait, and 3 ) the location of the loci controlling gene expression with respect to the position of the gene itself (cis- or trans- 
acting loci). A gene was assumed to be cis-regulated when the locus controlling its expression level mapped within $10 \mathrm{Mb}$ of the gene itself, and trans-regulated otherwise. Finally, results obtained using two different pre-processing methods were considered consistent when the distance between their linkage peaks was less than $20 \mathrm{Mb}$. Concordance rates between two or more methods were defined as the ratio of the number of consistent linkage peaks between methods over the total number of linkage peaks detected by any of the three methods.

\section{Results}

\section{Comparison of the linkage signals detected for the three methods}

Figure 1 gives the number of linkage signals for each preprocessing method in parentheses as well as the concordance rate between methods evaluated as described above. Not surprisingly, more linkage signals were found for the most heritable traits than for those with the highest variance. More linkage signals were detected with GCRMA than with the two other methods, especially in two sets, the set of 350 traits randomly chosen and that of 100 nonexpressed traits. In general, less than half of the linkage signals are concordant between two or three methods. However, these rates vary according to the group of genes analyzed: the most important discrepancies were observed for the set of non-expressed genes and the set of the 350 genes chosen at random: $74 \%$ and $63.7 \%$ of the signals, respectively, were specific to the GCRMA preprocessing method. Furthermore, for the group of 100 non-expressed genes, 2 signals with LOD $\geq 3$ were
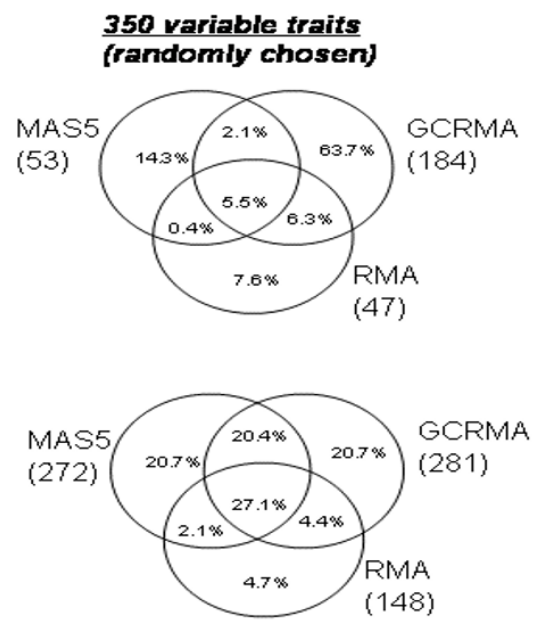

100 most heritable traits detected with MAS5, whereas 95 and 25 signals were detected with GCRMA and RMA, respectively. For the three other groups, RMA was the pre-processing method that generated the lowest rate of specific signals (from $4.7 \%$ to $11.3 \%)$.

\section{Comparison of the number of traits with at least one linkage signal as well as the number of linkage signals per trait}

Table 1 shows the number of detected eQTLs and the average number of linkage signals per trait. Again, on average, we observe that loci regulating gene expression were found for more genes when data were pre-processed using GCRMA, especially in two sets of traits. In the set of the 350 traits chosen at random, a high proportion of the linkage signals (64\%) were accounted by only two of the expression traits, SYMPK and ARG2. The same trend was observed in the set of non-expressed traits, where $60 \%$ of the signals were accounted by the two traits TIE1 and FUT7.

\section{Comparison of the cis-acting and trans-acting detected eQTLs}

The number of cis-, cis-trans, and trans-acting loci detected by each method is given in Table 2. Similar distributions were obtained with all three pre-processing methods. The majority of the detected traits are trans-regulated: from $65-68 \%$ in the set of non-expressed genes, to $76-89 \%$ in the set of 350 variable traits. Nevertheless, for cis-regulating signals (results not shown), concordance rates between the three methods were $44 \%$ (4/9) for the group

100 non-expressed traits
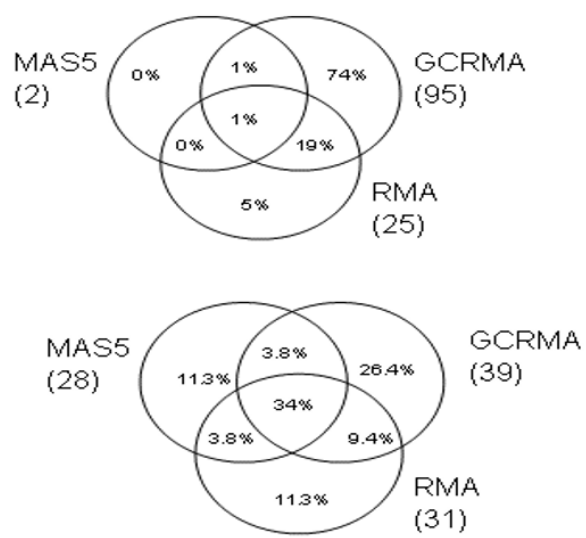

100 most variable traits

Figure I

Concordance and discordance rates of the linkage signals between pre-processing methods (with number of linkage signals per method). 
Table I: Number of detected traits (having at least one linkage signal) and mean number of linkage signals per trait

\begin{tabular}{|c|c|c|c|c|c|c|}
\hline \multirow[b]{2}{*}{ Set of traits } & \multicolumn{3}{|c|}{ No. Traits detected (\%) } & \multicolumn{3}{|c|}{ Mean no. of linkage signals by detected trait (range) } \\
\hline & MAS5 & GCRMA & RMA & MAS5 & GCRMA & RMA \\
\hline 350 random & $37 / 350(11 \%)$ & $45 / 350(13 \%)$ & $36 / 350(10 \%)$ & $1.43(1-4)$ & $4.08(I-6 I)$ & $1.30(1-3)$ \\
\hline 100 most variable & $18 / 100(18 \%)$ & $21 / 100(21 \%)$ & $17 / 100(17 \%)$ & $1.55(1-4)$ & $1.85(1-5)$ & $1.82(1-7)$ \\
\hline 100 most heritable & $39 / 100(39 \%)$ & $36 / 100(36 \%)$ & $34 / 100(34 \%)$ & $6.97(I-90)$ & $7.8(|-| I I)$ & $4.35(I-54)$ \\
\hline 100 non-expressed & $2 / 100(2 \%)$ & $14 / 100(14 \%)$ & $7 / 100$ (7\%) & $I(I-I)$ & $6.5(1-30)$ & $3.6(1-9)$ \\
\hline
\end{tabular}

of 350 traits chosen at random, 100\% (1/1) in the group of non-expressed genes, $73 \%(11 / 15)$ in the group of the most heritable genes, and $60 \%(3 / 5)$ in the group of the most variable traits. Further, concordance rates between GCRMA and RMA were especially high and always greater than $75 \%$. On the other hand, in the remaining set of signals (trans-regulators), much lower concordance rates were obtained ( $<34 \%$, for all sets of genes studied).

\section{Discussion}

As previously suggested by other studies $[6,8,10]$, our results confirm that pre-processing methods may also affect linkage outcomes. However, this impact depends on the way traits were selected for genetic analysis. Choosing the traits on the basis of a high heritability value led to a minimum discrepancy between methods. Conversely, discrepancies were more important in the group of nonexpressed traits or in the group of variable traits chosen at random (note that in this last group, heritability ranges

Table 2: Number of cis-, cis/trans, and trans-regulated traits for each method (proportion among traits having at least one linkage signal)

\begin{tabular}{llll}
\hline & cis & cis/trans & trans \\
\hline $\begin{array}{l}\text { 350 variable traits } \\
\text { MAS5 }\end{array}$ & $7(19 \%)$ & $2(5 \%)$ & $28(76 \%)$ \\
GCRMA & $4(9 \%)$ & $1(2 \%)$ & $40(89 \%)$ \\
RMA & $3(8 \%)$ & $1(3 \%)$ & $32(89 \%)$ \\
\hline I00 most variable traits & & & \\
MAS5 & $1(50 \%)$ & $0(0 \%)$ & $1(50 \%)$ \\
GCRMA & $1(7 \%)$ & $0(0 \%)$ & $13(93 \%)$ \\
RMA & $1(14 \%)$ & $0(0 \%)$ & $6(86 \%)$ \\
\hline I00 most heritable traits & & & \\
MAS5 & $2(11 \%)$ & $3(17 \%)$ & $13(72 \%)$ \\
GCRMA & $2(9.5 \%)$ & $2(9.5 \%)$ & $17(81 \%)$ \\
RMA & $3(18 \%)$ & $1(6 \%)$ & $13(76 \%)$ \\
\hline I 00 non-expressed traits & & & \\
MAS5 & $8(20 \%)$ & $6(15 \%)$ & $25(65 \%)$ \\
GCRMA & $7(19 \%)$ & $5(14 \%)$ & $24(67 \%)$ \\
RMA & $9(26 \%)$ & $2(6 \%)$ & $23(68 \%)$ \\
\hline
\end{tabular}

from $0 \%$ to $50 \%$ ). Furthermore, we noticed that the three pre-processing methods agree much better for cis-acting than for trans-acting regulators.

Several factors may explain partly why the three methods produce different results. First, as already stated in the Background, the underlying models converting probe level data to expression values are different from one method to another. Although the normalization step is not the same for the three methods, previous work has shown that these differences have little effect relative to that of the background correction, which entails a variance/bias trade-off. Especially, it has been shown that background correction decreases the bias but that naïve background correction procedures, such as MAS5 and RMA, increase the variance [8]. GCRMA is supposed to provide a good balance between accuracy and precision by doing adequate non-specific binding correction. Our study suggests that the large impact of the background correction also applies to eQTL mapping results. Indeed, RMA and GCRMA differ only by the background correction step, yet their concordance rates were not particularly high. Interestingly, Irizarry et al. [8] observed that differences in precision between RMA and GCRMA were higher in the case of genes with low expression, with GCRMA giving the smallest bias. We also observed a greater discordance rate between RMA and GCRMA for the set of nonexpressed genes.

Another potential factor for explaining differences between methods is departure from normality of the phenotypic distribution, especially when using a variancecomponent approach. We found that, in general, GCRMA led to the highest rate of traits failing the Shapiro normality test. Among expressed genes, these rates were 5.4, 0.8, and $1.7 \%$ for GCRMA, RMA, and MAS5, respectively (using a Bonferroni correction for multiple testing at level $5 \%$ ). These rates might explain the large number of linkage signals observed for a few traits with GCRMA. However, it seems unlikely that conflicting eQTL mapping results are mainly due to differences in the gene expression distributions per se. 
In conclusion, the true genetic determinants of the studied traits in the GAW Problem 1 data are unknown, preventing us from drawing definite conclusions on the best and more robust pre-processing method. Further, in the context of a genome-scan, high agreement rates across experiments are not expected because most of the linkage signals are likely to be false positives. It is unclear whether it would be sound to use several pre-processing methods in a systematic manner. Such guidelines were proposed recently but remain controversial [10-12]. In our study, we found very poor agreement between pre-processing methods in the set of the non-expressed but most variable genes (i.e., our Set 4), suggesting that filtering genes on their detectable presence in the tissue of interest is also an important step. To filter genes not only on their variability but also on their presence in the tissue analyzed is one of our main messages.

\section{Competing interests}

The author(s) declare that they have no competing interests.

\section{Acknowledgements}

This article has been published as part of BMC Proceedings Volume I Supplement I, 2007: Genetic Analysis Workshop 15: Gene Expression Analysis and Approaches to Detecting Multiple Functional Loci. The full contents of the supplement are available online at http://www.biomedcentral.com/ $\underline{|753-656| / \mid \text { ? issue=SI. }}$.

\section{References}

I. Cheung VG, Conlin LK, Weber TM, Arcaro M, Jen KY, Morley M, Spielman RS: Natural variation in human gene expression assessed in lymphoblastoid cells. Nat Genet 2003, 33:422-425.

2. Cheung VG, Spielman RS, Ewens KG, Weber TM, Morley M, Burdick JT: Mapping determinants of human gene expression by regional and whole genome association. Nature 2005, 437: $1365-1369$.

3. Morley M, Molony CM, Weber T, Devlin JL, Ewens KG, Spielman RS, Cheung VG: Genetic analysis of genome-wide variation in human gene expression. Nature 2004, 430:743-747.

4. Affymetrix: Affymetrix Microarray Suite User Guide, version 4 Santa Clara, CA: Affymetrix, Inc.

5. Affymetrix: Statistical Algorithms Description Document Santa Clara, CA: Affymetrix, Inc; 2002.

6. Irizarry R, Hobbs B, Collin F, Beazer-Barclay YD, Antonellis KJ, Scherf $U$, Speed TP: Exploration, normalization, and summaries of high density oligonucleotide array probe level data. Biostatistics 2003, 4:249-264.

7. Wu Z, Irizarry RA, Gentleman R, Murillo FM, Spencer F: A model based background adjustment for oligonucleotide expression arrays. J Am Stat Assoc 2004, 99:909-918.

8. Irizarry R, Wu Z, Jaffee H: Comparison of Affymetrix GeneChip expression measures. Bioinformatics 2006, 22:789-794.

9. Zakharin SO, Kim K, Mehta T, Chen L, Barnes S, Scheirer KE, Parrish RS, Allison DB, Page GP: Sources of variation in Affymetrix microarray experiments. BMC Bioinformatics 2005, 6:2I 4 .

10. Williams RBH, Cotsapas CJ, Cowley MJ, Chan E, Nott DJ, Little PFR: Normalization procedures and detection of linkage signal in genetical-genomic experiments. Nat Genet 2006, 38:855-856.

II. Petretto E, Mangion J, Cook SA, Aitman TJ, Pravenec M, Schulz H, Fischer J, Hubner N: Reply to "Normalization procedures and detection of linkage signal in genetical-genomics experiments". Nat Genet 2006, 38:858-859.

12. Chesler EJ, Bystrykh L, de Haan G, Cooke MP, Su A, Manly KF, Williams RW: Reply to "Normalization procedures and detection of linkage signal in genetical-genomics experiments". Nat Genet 2006, 38:856-858.

13. Abecasis GR, Cherny SS, Cookson WO, Cardon LR: Merlin-rapid analysis of dense genetic maps using sparse gene flow trees. Nat Genet 2002, 30:97-10I.
Publish with BioMed Central and every scientist can read your work free of charge

"BioMed Central will be the most significant development for disseminating the results of biomedical research in our lifetime. " Sir Paul Nurse, Cancer Research UK

Your research papers will be:

- available free of charge to the entire biomedical community

- peer reviewed and published immediately upon acceptance

- cited in PubMed and archived on PubMed Central

- yours - you keep the copyright 